\title{
STUDY ON GREENHOUSE TEMPERATURE VARIABLE UNIVERSAL ADAPTIVE FUZZY CONTROL
}

\author{
Weizhong Yang, Qiaoxue Dong, Yiming Wang ${ }^{*}$ \\ College of Information and Electronic Engineering, China Agricultural University, 10083 \\ Beijing, China \\ * Corresponding author, Address: P.O. Box 63, China Agricultural University, 17 Tsinghua \\ East Road, Beijing, 100083, P. R. China, Tel: +86-10-62737824, Email: ym_wang@263.net
}

\begin{abstract}
Basic fuzzy controller (BFC) has been successfully adopted to control greenhouse environment temperature. Variable universal adaptive fuzzy controller (VUAFC) which can contracts or expands the universal of the fuzzy variables of the BFC by the value of response error has been developed to get better control performance. By analyzing the result of experiments, VUAFC improved the performance of the fuzzy controller greatly.
\end{abstract}

Keywords: fuzzy control, greenhouse temperature, variable universal

\section{INTRODUCTION}

Suitable environment temperature is very important for the greenhousecrop. Fuzzy control is successful to use on control of the greenhouse environment temperature (Yang et al., 1999). As the nature of greenhouse temperature dynamic process is time-variance, the fuzzy controller must have the capability of adaptivity. VUAFC can adjust the universes of some fuzzy variables by the change of error. It will be higher on the control precise.

In the paper, firstly, the design of VUAFC of greenhouse environment temperature is introduced. Then, some experiments are carried out, and the control effects of BFC and VUAFC are compared.

Yang, W., Dong, Q. and Wang, Y., 2008, in IFIP International Federation for Information Processing, Volume 259; Computer and Computing Technologies in Agriculture, Vol. 2; Daoliang Li; (Boston: Springer), pp. 1417-1420. 


\section{DESIGN OF VUAFC OF GREENHOUSE ENVIRONMENT TEMPERATURE}

Variable universe (Li et al., 2002) means that some universes such as $X$, can change with changing variables $x$, denoted by

$$
X(x)=[-\alpha(x) E, \alpha(x) E]
$$

where $\alpha(x)$ are contraction-expansion factors of the universe $X$. Being relative to variable universes, the original universes $X=[-E, E]$ are called initial universes. The situation of variable universes changing is shown as Figure 1.

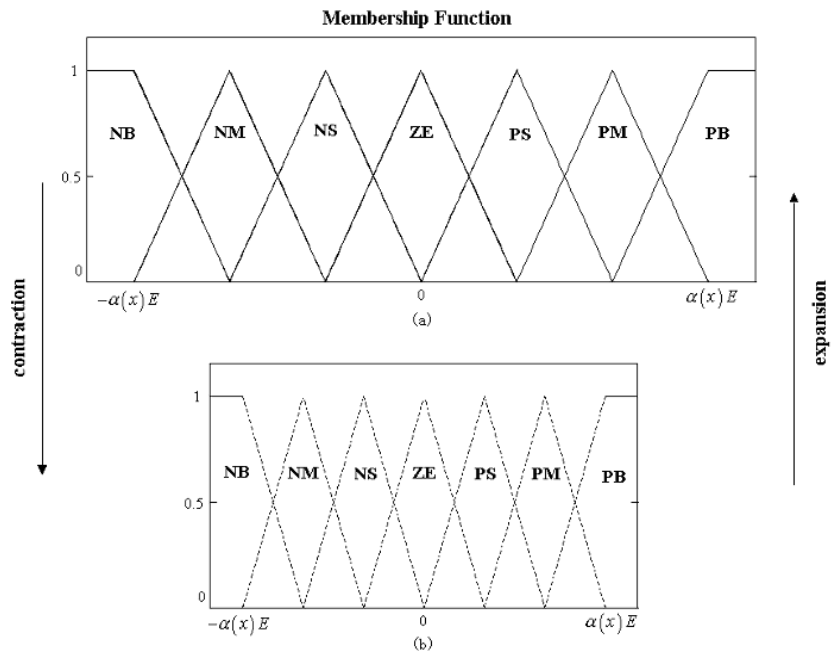

Figure 1. The schematic of contracting/expanding universe

In the paper, $\alpha(x)$ is as follow:

$$
\alpha(x)=\frac{1-\varepsilon e^{-k x^{2}}}{1-\varepsilon e^{-k E^{2}}} \text { and } k>0
$$

where $k$ is a parameter that can be chosen by designers, and $\varepsilon$ is a very small constant. Set $\varepsilon=0.8, k=1$.

\section{EXPERIMENTS AND ANALYSIS}

In order to evaluate the system capability roundly, three kinds of characteristics were applied. They are dynamic performance characteristics, stable-state performance characteristics and synthetic performance 
characteristics. The characteristics adopted in this paper are: overshoot, denoted by $\sigma ; 5 \%$ rise time, denoted by $5 \% t_{s}$; steady-state error, denoted by $e_{s s}$ and MITAE (Integral of product of Time and Absolute-value of Error) criterion (Xiang, 1986), denoted by $J_{\text {MITAE}}$.

MITAE criterion is denoted by

$$
J_{\text {MITAE }}=\frac{1}{t_{1}-t_{0}} \int_{t_{0}}^{t_{1}} t|e(t)| d t
$$

In the formula above, the time interval from $t_{0}$ to $t_{1}$ is the transient time, And $e(t)$ is the static error of the system response.

From (3), $J_{\text {MITAE }}$ is the time weighting sum of system error. Since error of steady system reduces gradually as time elapses, it can be concluded that $J_{\text {MITAE }}$ can reflect the dynamic and static performance of system. The smaller value of $J_{\text {MITAE }}$ can indicate quick response speed, short regulation time, small over-regulation and small steady-state error. When $J_{\text {MITAE }}$ reaches minimum, dynamic process of system response is optimized. It is very suitable to use this index to evaluate the dynamic response performance of greenhouse environmental temperature.

The chamber temperature control experiments were carried out in the greenhouse located in China Agriculture University in Dec. 2003. During the experiment period some tomato plants were planted in the greenhouse, and began to be harvested. Because air temperature is low in the winter night, heater is needed to maintain the growth of tomato; the experiment is executed during the night. The chamber temperature set point is 13 degrees Celsius. The chamber temperature depends on the hot water flow rate of the heating system, and the flow rate is related to the valve travel. The supervisory computer measures the chamber temperature and calculates $e_{1}$ and $e_{2}$, the inputs of the controller then, infer $u$, the output of the controller. $u$ is the time that the valve is powered, if $u>0$ the valve position augments and vice versa.

The course of the experiment was that the BFC was adopted firstly, the parameters was $k_{1}=2, k_{2}=20$ and $k_{3}=30$, the response curve was logged. Then the VUAFC was applied, and the response performances were measured. Table 1 lists the performances of the 2 fuzzy controllers; they are overregulation $(\sigma), 5 \%$ rise time $(5 \% t s)$, steady-state error $\left(e_{s s}\right)$, and $J_{\text {MITAE }}$. The Figure 2 shows the response curves of them.

Table 1. Performances of $\mathrm{BFC}$ and VUAFC

\begin{tabular}{llll|l}
\hline & $\sigma(\%)$ & $5 \% t_{s}(\min )$ & $e_{i s}$ & $J_{\text {MSIAZ }}$ \\
\hline BFC & 6.03 & 252 & 0.27 & 117.72 \\
VUAFC & 2.75 & 55 & 0.04 & 31.05 \\
\hline
\end{tabular}




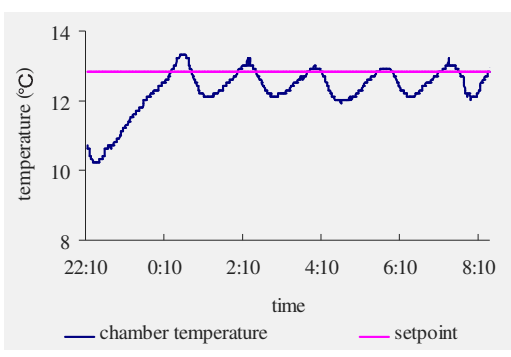

(a) the response curve of $\mathrm{BFC}$

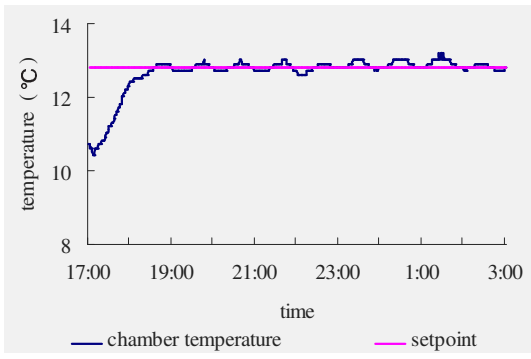

(b) the response curve of VUAFC

Figure 2. The response curves of 3 fuzzy controllers

\section{CONCLUSION}

VUAFC can improve the performance of environmental temperature control efficiently. Since it doesn't occupy too much resource, it can not only be used on normal computer, but also be used on single-chip microcomputer system as well.

\section{ACKNOWLEDGEMENTS}

This study was supported by National Tenth-Five year-Plan of China (Grant (Grant No. 2001BA503B01).

\section{REFERENCES}

Li Hongxing, et al. Variable universe stable adaptive fuzzy control of nonlinear system. Science in China. Ser. E., 2002, 45: 225-240.

Xiang Guobo, Optimal control based on ITAE. Mechanical Industry Press, Beijing, 1986.

Yang Weizhong, et al., Experiment and research of greenhouse automatic control. Trans. of the CSAE, 15 259-261 (1999). 